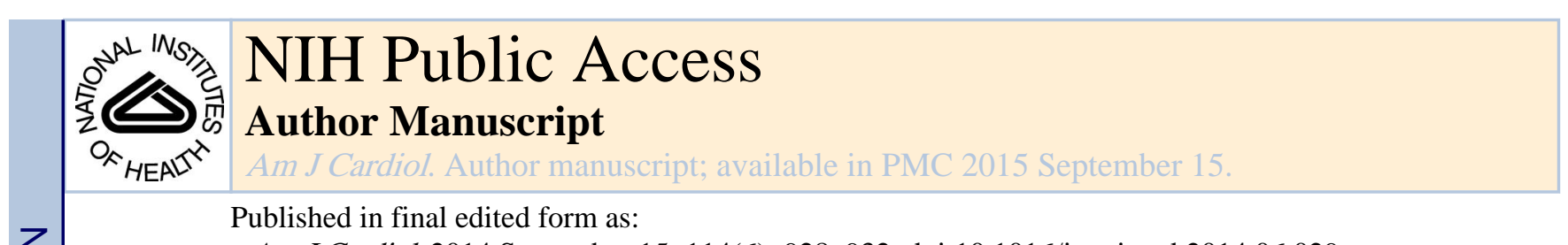

Published in final edited form as:

Am J Cardiol. 2014 September 15; 114(6): 928-932. doi:10.1016/j.amjcard.2014.06.029.

\title{
Family History as a Risk Factor for Peripheral Arterial Disease
}

\author{
Mahyar Khaleghi, MDa, lyad N. Isseh, MBBS ${ }^{a}$, Kent R. Bailey, PhD $^{b}$, and Iftikhar J. Kullo, \\ MDa \\ aDivision of Cardiovascular Diseases and the Gonda Vascular Center, Mayo Clinic, Rochester, \\ MN
}

bDivision of Biomedical Statistics and Informatics, Mayo Clinic, Rochester, MN

\begin{abstract}
The association of family history of peripheral arterial disease (PAD) with presence of PAD is largely unknown. We conducted a case-control study of 2296 patients with PAD $(69 \pm 10$ y, 64\% men) and 4390 controls (66 \pm 11 y, 62\% men) identified from non-invasive vascular and stress testing laboratories at Mayo Clinic, Rochester, Minnesota from October 2006 through June 2012. PAD was defined as a resting/post-exercise ankle-brachial index (ABI) $₫ 4.9$, a history of lower extremity revascularization, or having poorly compressible leg arteries. Controls were patients with normal ABI or without history of PAD. Family history of PAD was defined as having at least one first-degree relative who had undergone revascularization or stent placement for PAD before age 65. Logistic regression analyses were used to evaluate whether family history of PAD was associated with presence of $\mathrm{PAD}$, independent of conventional risk factors.

Family history of PAD was present more often in PAD patients than in controls with a resulting odds ratio (OR) (95\% confidence interval) of 2.20 (1.82-2.67). The association remained significant after adjustment for conventional risk factors; OR: 1.97 (1.60-2.42). The association was stronger in younger individuals (age <68 y); adjusted OR: $2.46(1.79-3.38)$ than in older individuals; adjusted OR: 1.61 (1.22-2.12). A greater number of affected relatives with PAD was also associated with higher odds of presence of PAD; adjusted OR: 1.86 (1.48-2.33) and 2.56 (1.60-4.11) for patients with 1 and $\geq 2$ affected relatives with PAD, respectively. In conclusion, individuals with family history of PAD have nearly double the odds of having PAD relative to those without such history.
\end{abstract}

\section{Keywords}

family history; risk factors; peripheral arterial disease

\footnotetext{
(C) 2014 Excerpta Medica, Inc. All rights reserved.

Correspondence: Iftikhar J. Kullo, M.D., Division of Cardiovascular Diseases, Mayo Clinic, 200 First Street Southwest, Rochester, MN 55905, FAX: 507-266-1617; TEL: 507-266-3964, kullo.iftikhar@mayo.edu.

Publisher's Disclaimer: This is a PDF file of an unedited manuscript that has been accepted for publication. As a service to our customers we are providing this early version of the manuscript. The manuscript will undergo copyediting, typesetting, and review of the resulting proof before it is published in its final citable form. Please note that during the production process errors may be discovered which could affect the content, and all legal disclaimers that apply to the journal pertain.
} 


\section{Introduction}

Family history is a simple yet powerful clinical tool for improving risk assessment and thereby prevention of common chronic diseases. ${ }^{1,2}$ Although multiple studies have shown that a family history of coronary heart disease (CHD) is a significant risk factor for $\mathrm{CHD},{ }^{3-5}$ few studies ${ }^{6,7}$ have assessed whether family history of PAD is a risk for factor for PAD. Small sample size and lack of power were major limitations of these studies ${ }^{6,7}$ and family history as a risk factor for PAD has not been examined in a large cohort of patients with PAD. We therefore investigated whether a family history of PAD or CHD was associated with presence of PAD in a large case-control study. We hypothesized that: i) a family history of PAD is a stronger risk factor for PAD than family history of CHD; ii) sibling history of PAD/CHD is a stronger risk factor than parental history because of greater sharing of environmental factors between siblings.

\section{Methods}

In October 2006, a biorepository of plasma and DNA of PAD patients and controls was initiated by recruiting patients referred for lower extremity arterial evaluation to the Mayo Clinic non-invasive vascular laboratory and patients referred to the stress ECG laboratory to screen for CHD. Between October 2006 and June 2012, 10,206 patients were recruited. All participants gave their written consent for participation in the studies and the use of their data for future research. The study protocol was approved by the Institutional Review Board of the Mayo Clinic.

We defined PAD as having an $\mathrm{ABI}$ of $₫ 0.9$ at rest or 1 min after exercise; or the presence of poorly compressible arteries (defined as an average $\mathrm{ABI}$ in one leg $>1.4$; or lower extremity BP of $\geq 255 \mathrm{~mm} \mathrm{Hg}$ ); or presence of two or more codes from a set of appropriate International Classification of Diseases, Ninth revision, Clinical Modification (ICD-9-CM) codes and a set of appropriate Current Procedural Terminology, 4th revision (CPT-4) codes. Possible PAD was defined as having only one code from the same set of appropriate ICD-9/ CPT-4 codes. The medical records of patients with possible PAD were manually reviewed by a trained physician (I.I.) to confirm their diagnosis. Patients who did not have PAD (presence of normal ABI or absence of the same set of appropriate ICD-9/CPT-4 PAD codes) were classified as controls (Fig. 1). We excluded 144 patients who withdrew from the study after initial recruitment and those who identified themselves as adopted $(\mathrm{n}=112)$.

Several non-atherosclerotic vascular diseases can result in a low ABI, thereby mimicking atherosclerotic PAD; these include several vasculitides, Buerger's disease, embolism, trauma to leg arteries and other rare arteriopathies. Patients who had an abnormal ABI secondary to these conditions $(\mathrm{n}=85)$ were excluded as well.

Family history of PAD was obtained using a questionnaire given to the patients at the time of recruitment; 7027 patients $(69 \%)$ returned the questionnaire. From this cohort we identified 2296 PAD cases and 4390 controls (Fig.1). Participants were asked about presence of PAD and CHD in first-degree relatives, including mother, father, full siblings, sons, and daughters. Family history of PAD was considered to be present if a first-degree relative underwent lower extremity revascularization or stent placement before age 65 . 
Family history of CHD was considered to be present if a first-degree relative had myocardial infarction or coronary revascularization or stent placement before age 65 .

Specific data elements of potential relevance to PAD were abstracted from the electronic medical record (EMR). These include birth date, sex and race. Height, weight and body mass index (BMI) closest to the index date (defined as the date of vascular laboratory evaluation or stress ECG testing at the time of recruitment) were also abstracted from the EMR directly.

Smoking status was ascertained from the study questionnaire and 'ever' smoking defined as having smoked more than 100 cigarettes. To ascertain other risk factors and comorbidities we used previously validated electronic algorithms that included ICD-9-CM codes, medication use and laboratory data in the EMR. ${ }^{8}$ The presence of relevant ICD-9-CM codes up to six months following the index date was considered. Total, high-density lipoprotein, and low-density lipoprotein cholesterol, triglycerides, fasting blood sugar and glycosylated hemoglobin were obtained from the laboratory database using a window of one year around the time of patient enrollment. Resting systolic and diastolic BP were obtained as structured observations from the EMR. The diagnosis of hypertension was established based on two BP readings of $\geq 140 / 90 \mathrm{~mm} \mathrm{Hg}$ within three months closest to the index date, or a previous diagnosis of hypertension and current treatment with antihypertensive medication. Similarly, diabetes was diagnosed if a patient had fasting plasma glucose $\geq 126 \mathrm{mg} / \mathrm{dl}$, or a random glucose $>200 \mathrm{mg} / \mathrm{dl}$, or hemoglobin A1c of $>6.5 \%$, or had a previous diagnosis and was on treatment with oral hypoglycemic agent(s) or insulin. Dyslipidemia was defined as total cholesterol $>220 \mathrm{mg} / \mathrm{dl}$, or high-density lipoprotein cholesterol $<40 \mathrm{mg} / \mathrm{dl}$ (in men), $<45$ $\mathrm{mg} / \mathrm{dl}$ (in women), or triglycerides $>200 \mathrm{mg} / \mathrm{dl}$ or the use of a lipid-lowering medication.

Continuous data are summarized as either mean $\pm \mathrm{SD}$ or median and quartiles. Between group differences were assessed by an unpaired two-tailed student's t-test or Wilcoxon rank sum test. Categorical data were expressed as percentages and between group differences were assessed by the chi-square test statistic. We constructed multivariable logistic regression models that adjusted for conventional risk factors and other potential confounding variables to assess whether family history of PAD or CHD was independently associated with PAD. Adjustments were performed for age and sex, BMI, smoking history, diabetes, hypertension, and dyslipidemia. In order to compare the association of a parental and sibling history of PAD and CHD with presence of PAD, we stratified study participants based on family history (parental or sibling history) and repeated analyses described above for each group separately. We also checked for interactions between conventional risk factors and family history in the prediction of PAD. A two-sided $P$-value of $<0.05$ was deemed statistically significant. Statistical analyses were carried out using the SAS v 9.1 (SAS Institute, Cary NC) software package.

\section{Results}

Participant characteristics are shown in Table 1. Patients with PAD were older and the proportion of men was similar in both groups. Patients with PAD had a higher prevalence of increased BMI, dyslipidemia, diabetes, history of smoking, and hypertension (Table 1). 
Prevalence of family history of PAD was significantly higher in patients with PAD than in controls (10.4\% vs. $5.0 \%, P<0.0001)$ (Table 2$)$. Both sibling and parental history of PAD were more often presented in PAD patients than controls ( 7.3 vs. $3.4 \%, P<0.0001$, and 2.9 vs. $1.3 \%, P<0.0001$, respectively). In univariable logistic regression analysis, family history of PAD was associated with higher OR: 2.20 (1.82-2.67) of having PAD. Multivariable logistic regression analysis showed that the association of family history of PAD with having PAD was only modestly attenuated and remained significant after adjustment for age, sex, BMI, ever smoking, diabetes, hypertension and dyslipidemia; OR: 1.97 (1.60-2.42) (Fig. 2A). Similar results were observed for sibling and parental history of PAD; OR: 1.86 $(1.46-2.38)$ and $2.30(1.56-3.39)$, respectively. We noted a significant $(P<0.01)$ interaction between age and family history of PAD in predicting presence of PAD. The interaction indicated that the association between family history and presence of PAD was stronger in younger subjects. Therefore, we also performed subgroup analyses stratifying by the median age $(68 \mathrm{y})$ of the entire cohort. The associations were stronger in the younger age group (Fig. 2A). The adjusted ORs of having PAD were 2.46 (1.79-3.38) and 1.61 (1.22-2.12), in the younger and older group, respectively.

The prevalence of family history of CHD was higher in patients with PAD than in controls (47.6\% vs. $39.5 \%, P<0.0001)$ (Table 3 ). In univariable logistic regression analysis, family history of CHD was associated with higher OR of having PAD: 1.39 (1.26-1.54). Similar results were observed for sibling history of CHD; OR: 1.58 (1.41-1.77). However, the association between parental history of CHD and presence of PAD was not statistically significant; OR: 1.09 (0.97-1.22). After adjustment for the above mentioned covariates, multivariable logistic regression analysis showed that the association of family history of CHD with PAD remained significant; OR: 1.25 (1.12-1.40) (Fig. 2B). We noted a significant $(P<0.01)$ interaction between age and family history of CHD in predicting presence of PAD. Subgroup analyses stratifying by the median age ( $68 \mathrm{y})$ showed the association was stronger in the younger age group; OR: 1.44 (1.21-1.71), with the older age group being only marginally associated with presence of PAD; OR: 1.13 (0.98-1.31) (Fig 2B).

A greater number of affected relatives with PAD or CHD was associated with higher odds of presence of PAD (Fig. 3). For number of affected relatives with PAD, in logistic regression models, the adjusted ORs were: $1.86(1.48-2.33)$ and $2.56(1.60-4.11)$ for patients with 1 and $\geq 2$ affected relatives with PAD, respectively (Fig 3A). The difference between the ORs was greater in the younger individuals and was not significant in older individuals. A greater number of affected relatives with CHD was also associated with higher odds of presence of PAD (Fig. 3B). The adjusted ORs were: $1.17(1.03-1.33)$ and $1.40(1.20-1.64)$ for patients with 1 and $\geq 2$ affected relatives with CHD, respectively. Similarly, the difference between the ORs was greater in the younger individuals but was marginally significant in the older individuals.

\section{Discussion}

The main findings of the present study are: i) family history of PAD was significantly associated with the presence of PAD, independent of conventional risk factors; ii) The 
association was stronger in individuals younger than $68 \mathrm{y}$ of age and in those with greater number of affected relatives; iii) parental and sibling history were similarly associated with PAD; iv) family history of CHD was also associated with presence of PAD, but the magnitude of the association was weaker than for family history of PAD. These findings suggest: i) shared genetic and environmental factors contribute to the risk of PAD and ii) the presence of genetic susceptibility variants shared between PAD and CHD as well as susceptibility variants that are unique to PAD.

Our findings extend in a robust manner earlier reports suggesting familial clustering of PAD. Valentine et $\mathrm{al}^{6}$ demonstrated that siblings $(\mathrm{n}=50)$ of patients with premature PAD had nearly 3-fold increased risk of occult disease in carotid, abdominal, and lower extremity territories on duplex ultrasound scanning, compared to controls. In the San Diego Population study, ${ }^{7}$ any family history of PAD or parental history of PAD was only marginally associated with presence of PAD and family history of cardiovascular disease was not associated with presence of PAD. This study was likely underpowered to detect significant associations as only 87 patients with PAD were included. In the population-based Swedish Twin Registry ${ }^{9}$ the odds ratio of having PAD in persons whose twin had PAD compared with persons whose twin did not have PAD was 17.7 (95\% CI, 11.7 to 26.6) for monozygotic twins and 5.7 (95\% CI, 4.1 to 7.9 ) for dizygotic twins. Moderate heritability has also been demonstrated for $\mathrm{ABI}$, a marker for PAD. ${ }^{10,11}$

We found that sibling and parental history of PAD confer similar odds for the presence of PAD. This is not consistent with the hypothesis that sibling history of PAD may be a stronger risk factor than parental history due to greater sharing of environmental factors between sibling pairs than between parent-offspring pairs. We did, however, find sibling history of CHD to be a stronger risk factor than parental history of CHD, consistent with several previous studies showing that individuals with a sibling history of early-onset CHD are at higher risk than those who have only parental history. ${ }^{12,13}$

The association between family history and presence of PAD was modified by age, being stronger in younger individuals. This is consistent with previous studies in which the attributed risk of family history of CHD was stronger among younger individuals. ${ }^{14}$ Genetic factors are more likely to manifest in younger than in older individuals. Aging and cumulative exposure to vascular risk factors may dilute the effect of genetic susceptibility variants thereby attenuating the effect of family history of PAD later in life. We did not find a significant interaction between family history and sex or any other conventional risk factors (diabetes, smoking, hypertension, or dyslipidemia) in predicting the presence of PAD.

We noted that the number of affected relatives with PAD was associated with the odds of prevalent PAD, especially in the younger participants; in our study individuals aged $<68 \mathrm{y}$ with $\geq 2$ affected relatives had an OR similar to that for smoking; $6.3(5.1-7.7)$ and higher than the OR associated with diabetes; 3.4 (2.7-4.2) or hypertension; $2.0(1.7-2.4)$. We observed a similar association between the number of affected relatives with CHD and prevalent PAD, but the magnitude of the association was weaker than for family history of PAD. To eliminate the potential bias from differences in family size between cases and 
controls, we adjusted the models for the size of family (number of all first degree relatives). Adjustment for family size did not change the strength of associations (analyses not shown).

The association of family history and presence of PAD could be due to familial aggregation of traditional risk factors. Although participants with PAD were slightly older and had higher prevalence of risk factors (diabetes, smoking, hypertension, and dyslipidemia) than controls, the association after adjustment for these risk factors. This finding suggests that the association of family history with PAD maybe due to genetic factors acting independent of conventional risk factors.

The strengths of this study include a large cohort of PAD cases and controls and assessment of family history based on an in-depth questionnaire. Limitations include: 1) an element of referral bias since the study population comprised of patients who were referred to Mayo Clinic. However, in a subset analysis of patients who were residents of Minnesota, Iowa, and Wisconsin ( $\mathrm{n}=4896$ ), we found similar results (analysis not shown); 2) our study sample consists predominantly of non-Hispanic whites $(>95 \%)$ and the extent to which our findings are generalizable to subjects of other ethnic backgrounds is not known; 3) we cannot exclude the presence of asymptomatic PAD in some of the control participants. However, this bias would tend to attenuate the association toward the null (underestimate the true associations; 4) as in other case-control studies, an element of recall bias cannot be exclude. Previous studies have shown that reported family history of CHD has a high sensitivity and specificity ${ }^{15-17}$ and most likely the recall bias is toward the null; ${ }^{18}$ 5) the response rate to the questionnaire was $69 \%$. Using the EMR, we were able to compare the characteristics of responders and non-responders. Responders were older but there was similar proportion of men and women in both groups. Responders had a slightly higher prevalence of diabetes, but the prevalence of other conventional risk factors were similar in the two groups (Supplement Table I).

\section{Supplementary Material}

Refer to Web version on PubMed Central for supplementary material.

\section{Acknowledgments}

Mahyar Khaleghi was supported by the National Institutes of Health (NIH) Vascular Medicine Fellowship Training Program K12 grant (HL083797). Iftikhar Kullo was supported by grant U01 HG-06379 from the National Human Genome Research Institute. This publication was made possible by Center for Translational Science Activities (CTSA) Grant Number UL1 TR000135 from the National Center for Advancing Translational Sciences (NCATS), a component of the NIH. Its contents are solely the responsibility of the authors and do not necessarily represent the official view of NIH.

\section{References}

1. Valdez R, Yoon PW, Qureshi N, Green RF, Khoury MJ. Family history in public health practice: a genomic tool for disease prevention and health promotion. Annu Rev Public Health. 2010; 31:69_ 87. 61 p following 87. [PubMed: 20070206]

2. Guttmacher AE, Collins FS, Carmona RH. The family history--more important than ever. N Engl J Med. 2004; 351:2333-2336. [PubMed: 15564550]

3. Shea S, Ottman R, Gabrieli C, Stein Z, Nichols A. Family history as an independent risk factor for coronary artery disease. J Am Coll Cardiol. 1984; 4:793-801. [PubMed: 6481018] 
4. Schildkraut JM, Myers RH, Cupples LA, Kiely DK, Kannel WB. Coronary risk associated with age and sex of parental heart disease in the Framingham Study. Am J Cardiol. 1989; 64:555-559. [PubMed: 2782245]

5. Pohjola-Sintonen S, Rissanen A, Liskola P, Luomanmaki K. Family history as a risk factor of coronary heart disease in patients under 60 years of age. Eur Heart J. 1998; 19:235-239. [PubMed: 9519316]

6. Valentine RJ, Guerra R, Stephan P, Scoggins E, Clagett GP, Cohen J. Family history is a major determinant of subclinical peripheral arterial disease in young adults. J Vasc Surg. 2004; 39:351356. [PubMed: 14743135]

7. Wassel CL, Loomba R, Ix JH, Allison MA, Denenberg JO, Criqui MH. Family history of peripheral artery disease is associated with prevalence and severity of peripheral artery disease: the San Diego population study. J Am Coll Cardiol. 2011; 58:1386-1392. [PubMed: 21920269]

8. Kullo IJ, Fan J, Pathak J, Savova GK, Ali Z, Chute CG. Leveraging informatics for genetic studies: use of the electronic medical record to enable a genome-wide association study of peripheral arterial disease. J Am Med Inform Assoc. 2010; 17:568-574. [PubMed: 20819866]

9. Wahlgren CM, Magnusson PK. Genetic influences on peripheral arterial disease in a twin population. Arterioscler Thromb Vasc Biol. 2011; 31:678-682. [PubMed: 21164079]

10. Kullo IJ, Turner ST, Kardia SL, Mosley TH Jr, Boerwinkle E, de Andrade M. A genome-wide linkage scan for ankle-brachial index in African American and non-Hispanic white subjects participating in the GENOA study. Atherosclerosis. 2006; 187:433-438. [PubMed: 16280126]

11. Murabito JM, Guo CY, Fox CS, D'Agostino RB. Heritability of the ankle-brachial index: the Framingham Offspring study. Am J Epidemiol. 2006; 164:963-968. [PubMed: 16928729]

12. Murabito JM, Pencina MJ, Nam BH, D'Agostino RB Sr, Wang TJ, Lloyd-Jones D, Wilson PW, O'Donnell CJ. Sibling cardiovascular disease as a risk factor for cardiovascular disease in middleaged adults. JAMA. 2005; 294:3117-3123. [PubMed: 16380592]

13. Nasir K, Michos ED, Rumberger JA, Braunstein JB, Post WS, Budoff MJ, Blumenthal RS. Coronary artery calcification and family history of premature coronary heart disease: sibling history is more strongly associated than parental history. Circulation. 2004; 110:2150-2156. [PubMed: 15466626]

14. Marenberg ME, Risch N, Berkman LF, Floderus B, de Faire U. Genetic susceptibility to death from coronary heart disease in a study of twins. N Engl J Med. 1994; 330:1041-1046. [PubMed: 8127331]

15. Bensen JT, Liese AD, Rushing JT, Province M, Folsom AR, Rich SS, Higgins M. Accuracy of proband reported family history: the NHLBI Family Heart Study (FHS). Genet Epidemiol. 1999; 17:141-150. [PubMed: 10414557]

16. Hastrup JL, Hotchkiss AP, Johnson CA. Accuracy of knowledge of family history of cardiovascular disorders. Health Psychol. 1985; 4:291-306. [PubMed: 4054077]

17. Murabito JM, Nam BH, D'Agostino RB Sr, Lloyd-Jones DM, O'Donnell CJ, Wilson PW. Accuracy of offspring reports of parental cardiovascular disease history: the Framingham Offspring Study. Ann Intern Med. 2004; 140:434-440. [PubMed: 15023709]

18. Silberberg JS, Wlodarczyk J, Fryer J, Ray CD, Hensley MJ. Correction for biases in a populationbased study of family history and coronary heart disease. The Newcastle Family History Study I. Am J Epidemiol. 1998; 147:1123-1132. [PubMed: 9645790] 


\section{Highlights}

- We conducted a large case-control study to investigate whether family history of peripheral arterial disease (PAD) and coronary heart disease (CHD) was associated with presence of PAD.

- We showed that family history of PAD and CHD are independently associated with presence of PAD, the former being more strongly associated.

- In our study, parental or sibling history of PAD conferred similar odds.

- We found that the association was age-dependent, being stronger in younger individuals and the magnitude of the association was greater in those with greater number of affected relatives. 


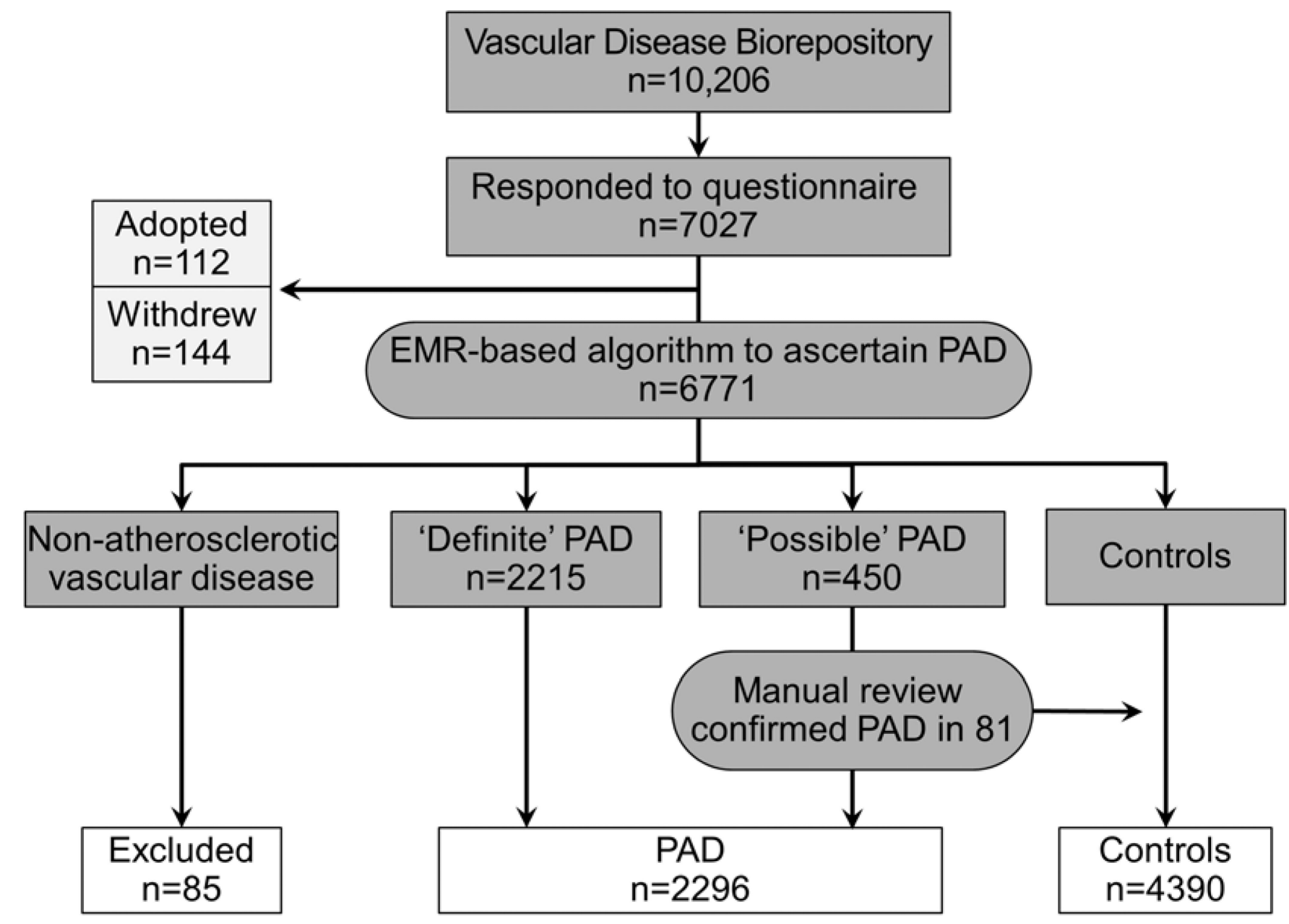

Figure 1.

Ascertainment of PAD cases and controls for this study. PAD: peripheral arterial disease. 


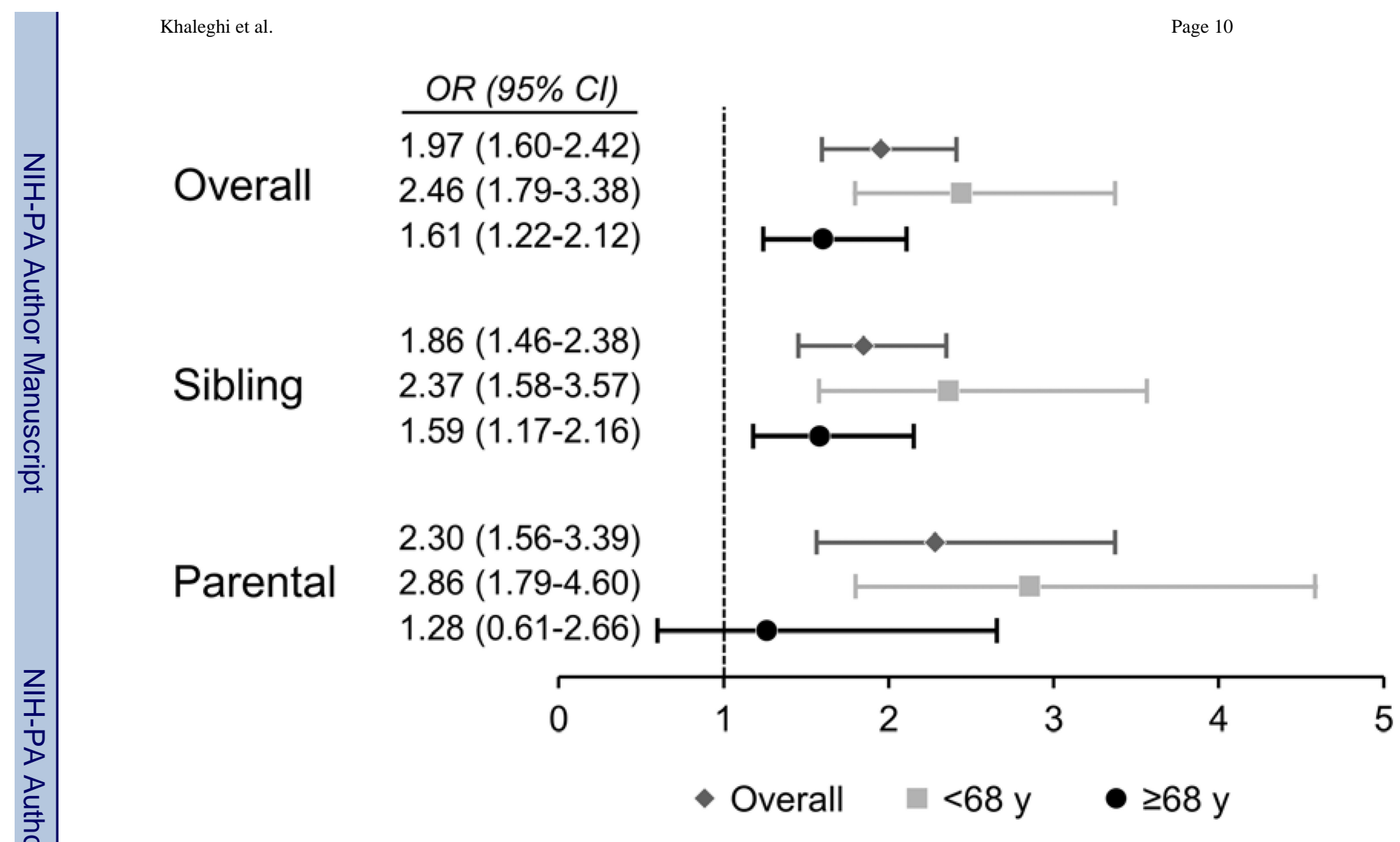




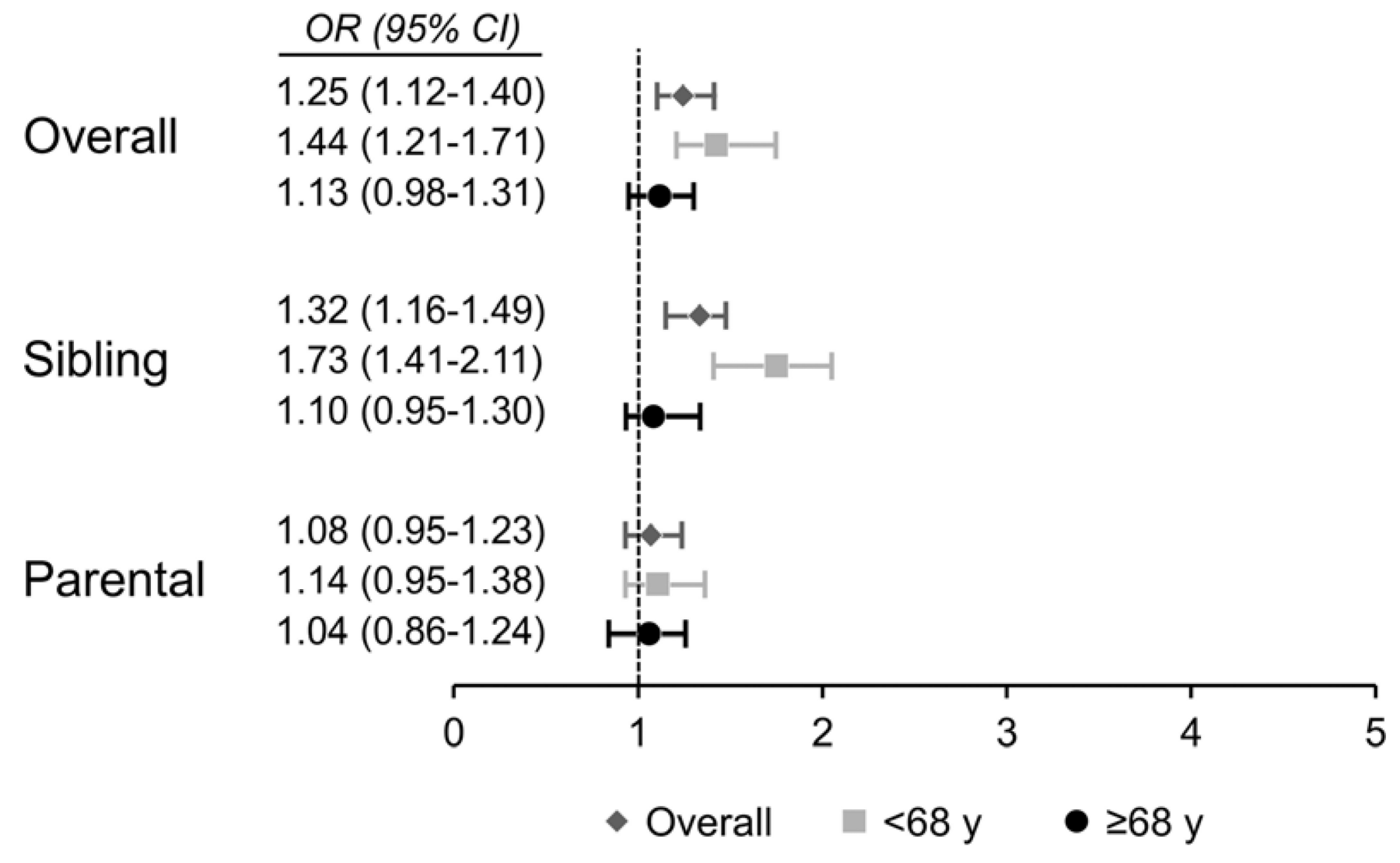

Figure 2.

Association of family history of PAD (A) and CHD (B) with presence of PAD. Odds ratios (95\% CI) from multivariate regression analysis are shown. Models were adjusted for age, sex, hypertension, diabetes, smoking, and body mass index. CHD: coronary heart disease; PAD: peripheral arterial disease. 


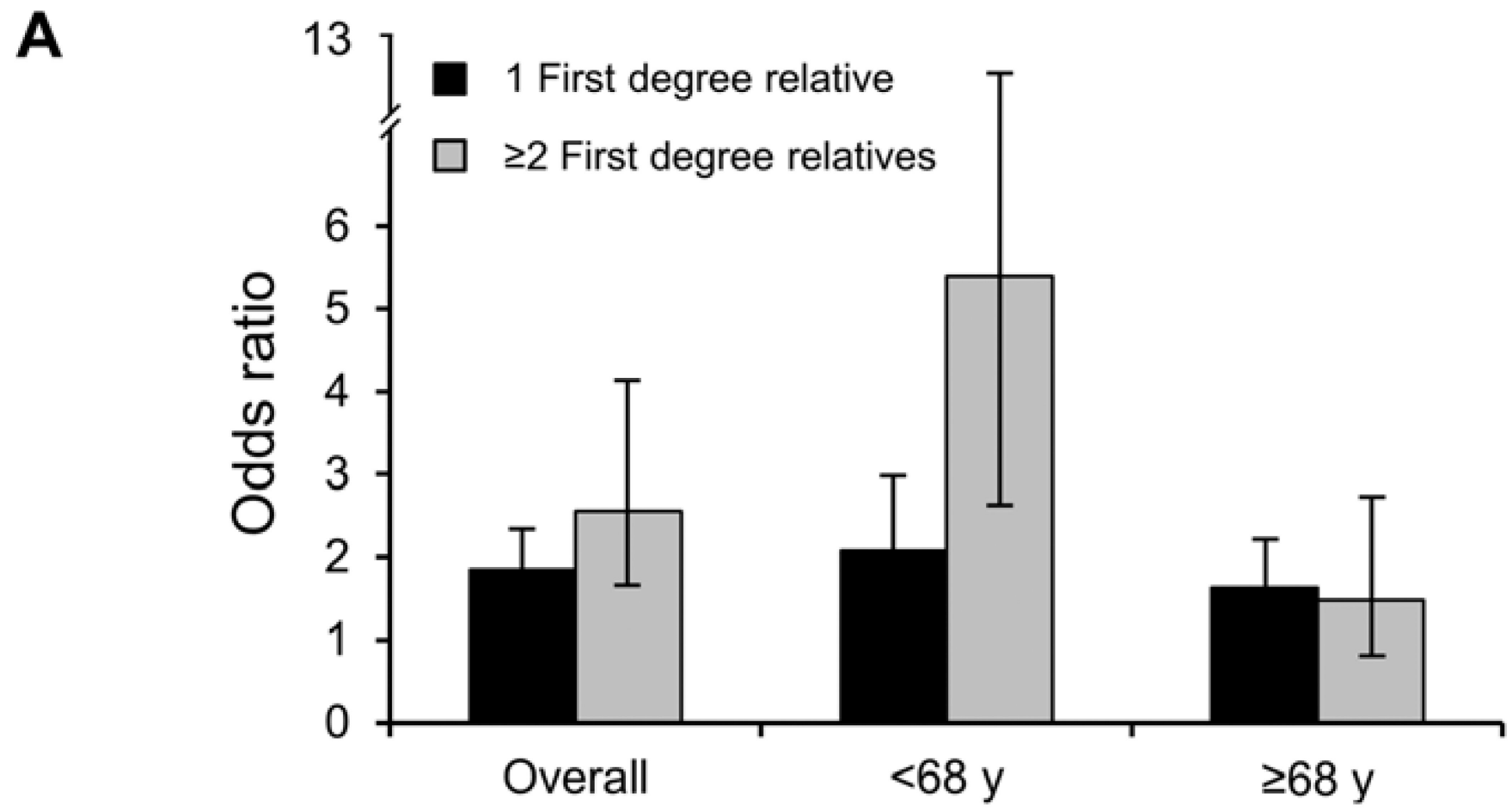

B

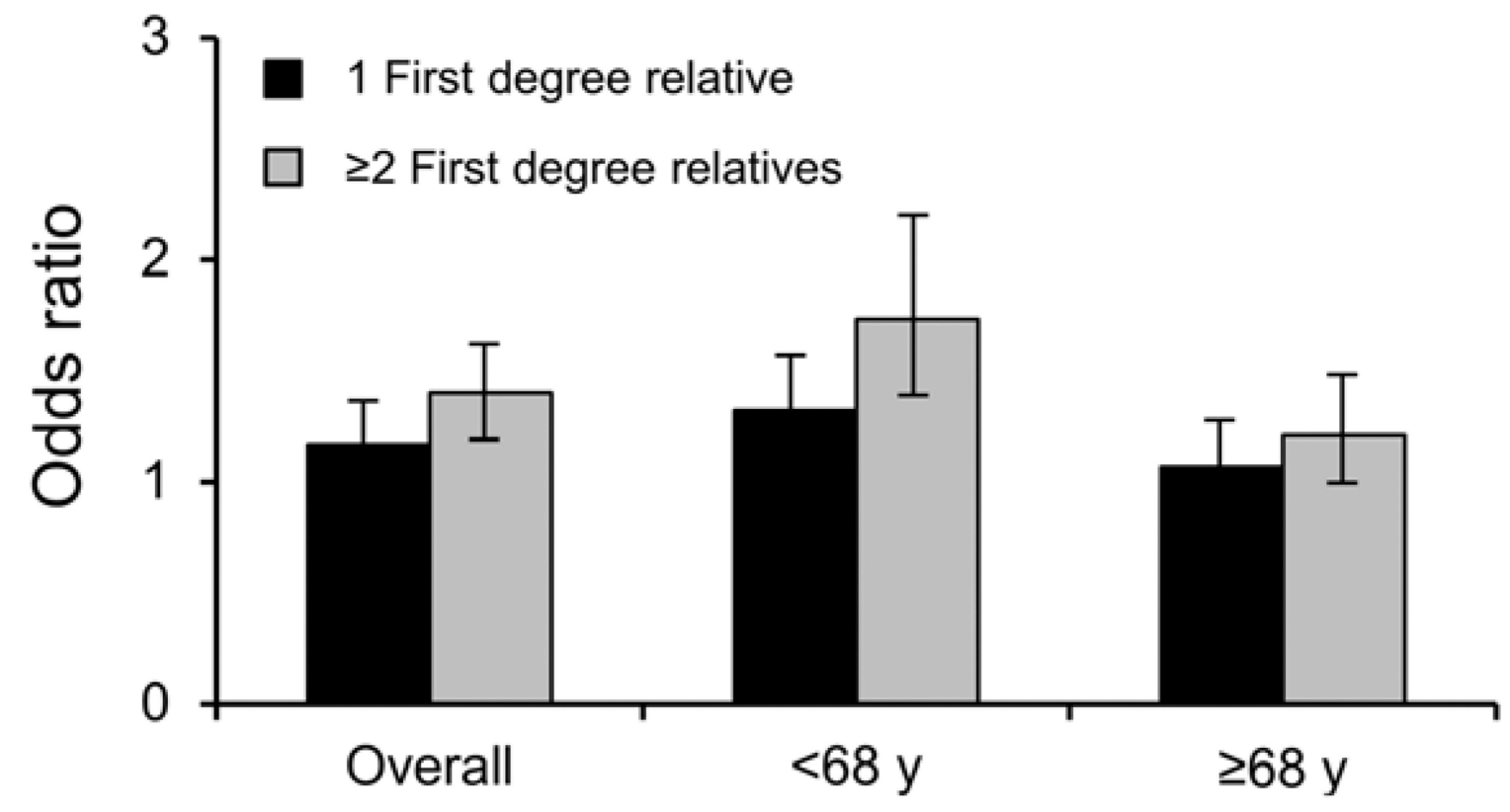

Figure 3.

Association of the number of affected relatives (A) and CHD (B) with presence of PAD. Odds ratios $(95 \% \mathrm{CI})$ from multivariate regression analysis are shown. Models were adjusted for age, sex, hypertension, diabetes, smoking, and body mass index. CHD: coronary heart disease; PAD: peripheral arterial disease. 


\section{Table 1}

Participant characteristics

\begin{tabular}{lccr}
\hline Variables & PAD $(\mathbf{n = 2 2 9 6})$ & Control $(\mathbf{n = 4 3 9 0})$ & $\boldsymbol{P}$ value \\
\hline Age (years) & $69.0 \pm 10.4$ & $66.2 \pm 11.1$ & $<0.001$ \\
Men & $1457(63.5 \%)$ & $2713(61.8 \%)$ & 0.18 \\
BMI $\left(\mathrm{kg} / \mathrm{m}^{2}\right)$ & $29.3 \pm 5.5$ & $28.7 \pm 5.3$ & $<0.001$ \\
Dyslipidemia & $1815(79.1 \%)$ & $3043(69.3 \%)$ & $<0.001$ \\
Diabetes mellitus & $834(36.3 \%)$ & $701(16.0 \%)$ & $<0.001$ \\
Ever smoked & $1841(80.2 \%)$ & $2197(50.0 \%)$ & $<0.001$ \\
Hypertension & $1517(66.1 \%)$ & $2077(47.3 \%)$ & $<0.001$ \\
Number of first degree relatives with PAD & & \\
0 & $2058(89.6 \%)$ & $4171(95.0 \%)$ & $<0.001$ \\
1 & $190(8.3 \%)$ & $184(4.2 \%)$ & \\
2 & $30(1.3 \%)$ & $22(0.5 \%)$ & \\
$\geq 3$ & $18(0.8 \%)$ & $13(0.3 \%)$ & \\
Number of first degree relatives with CHD & & \\
0 & $933(40.6 \%)$ & $1934(44.1 \%)$ & $<0.001$ \\
1 & $706(30.7 \%)$ & $1497(34.1 \%)$ & \\
2 & $405(17.6 \%)$ & $633(14.4 \%)$ & \\
\hline 3 & $252(11.0 \%)$ & $326(7.4 \%)$ & \\
\hline
\end{tabular}

Continuous variables are presented as means \pm standard deviation, whereas categorical variables are presented as counts and percentages. BMI: body mass index; CHD: coronary heart disease; PAD: peripheral arterial disease. 


\section{Table 2}

Univariable associations of family history of peripheral arterial disease with presence of peripheral arterial disease

\begin{tabular}{lccc}
\hline & PAD $(\mathbf{n = 2 2 9 6})$ & Control (n=4390) & OR (95\% CI) \\
\hline Family history of PAD & $238(10.4 \%)$ & $219(5.0 \%)$ & $2.20(1.82-2.67)$ \\
Sibling history of PAD & $168(7.3 \%)$ & $151(3.4 \%)$ & $2.22(1.77-2.78)$ \\
Parental history of PAD & $66(2.9 \%)$ & $61(1.3 \%)$ & $2.10(1.48-2.99)$ \\
\hline Younger (individuals $<\mathbf{6 8} \mathbf{y})$ & PAD (n=941) & Control (n=2362) & OR (95\% CI) \\
\hline Family history of PAD & $112(11.9 \%)$ & $101(4.3 \%)$ & $3.02(2.28-4.00)$ \\
Sibling history of PAD & $68(7.2 \%)$ & $56(2.4 \%)$ & $3.21(2.23-4.61)$ \\
Parental history of PAD & $51(5.4 \%)$ & $44(1.9 \%)$ & $3.02(2.00-4.55)$ \\
\hline Older (individuals $\mathbf{~} \mathbf{6 8} \mathbf{y})$ & PAD (n=1355) & Control (n=2028) & OR (95\% CI) \\
\hline Family history of PAD & $126(9.3 \%)$ & $118(5.8 \%)$ & $1.66(1.28-2.15)$ \\
Sibling history of PAD & $100(7.4 \%)$ & $95(4.7 \%)$ & $1.62(1.21-2.17)$ \\
Parental history of PAD & $15(1.1 \%)$ & $17(0.8 \%)$ & $1.32(0.66-2.66)$ \\
\hline
\end{tabular}

PAD: peripheral arterial disease. 
Table 3

Univariable associations of family history of coronary heart disease with presence of peripheral arterial disease

\begin{tabular}{lccc}
\hline & PAD (n=2296) & Control (n=4390) & OR (95\% CI) \\
\hline Family history of CHD & $1093(47.6 \%)$ & $1733(39.5 \%)$ & $1.39(1.26-1.54)$ \\
Sibling history of CHD & $710(30.9 \%)$ & $968(22.0 \%)$ & $1.58(1.41-1.77)$ \\
Parental history of CHD & $576(25.1 \%)$ & $1033(23.5 \%)$ & $1.09(0.97-1.22)$ \\
\hline Younger (individuals $<\mathbf{6 8} \mathbf{y})$ & PAD (n=941) & Control (n=2362) & OR (95\% CI) \\
\hline Family history of CHD & $563(59.8 \%)$ & $1303(55.2 \%)$ & $1.65(1.42-1.92)$ \\
Sibling history of CHD & $279(29.6 \%)$ & $389(16.5 \%)$ & $2.14(1.79-2.55)$ \\
Parental history of CHD & $299(31.8 \%)$ & $640(27.1 \%)$ & $1.25(1.06-1.48)$ \\
\hline Older (individuals $\mathbf{~} 68 \mathbf{y})$ & PAD (n=1355) & Control (n=2028) & OR (95\% CI) \\
\hline Family history of CHD & $800(59.0 \%)$ & $1153(56.9 \%)$ & $1.19(1.03-1.36)$ \\
Sibling history of CHD & $431(31.8 \%)$ & $579(28.6 \%)$ & $1.17(1.01-1.36)$ \\
Parental history of CHD & $277(20.4 \%)$ & $393(19.4 \%)$ & $1.07(0.90-1.27)$ \\
\hline
\end{tabular}

CHD: coronary heart disease; PAD: peripheral arterial disease. 